\title{
The Impact of Ergonomic Design on Smart Garments
}

\author{
Rachel S. Boldt ${ }^{1}$, Luisa M. Arruda ${ }^{1}$, Yao Yu ${ }^{1}$, Helder Carvalho ${ }^{1}$, Miguel A. F. Carva- \\ lho ${ }^{1}$, and Fernando B. N. Ferreira ${ }^{1}$ \\ ${ }^{1}$ 2C2T - Centro de Ciência e Tecnologia Têxtil, University of Minho, Guimaraes, Portugal \\ r.boldt@det.uminho.pt/rachelsagerb@gmail.com
}

\begin{abstract}
.
This paper reports the design process of a smart garment, which comprised 3lead sEMG (Surface Electromyography) electrodes. The ergonomic design is central for a proper monitoring response because it is a related with the stability and very well contacted between the electrode and the user' body. For this, different body postures and the t-shirt behavior on the body was studied and simulated using a virtual prototype. This approach contributed to understanding ways to solving problems related to fit and the electrodes' stabilization. Furthermore, physical and electronic tests using a prototype on a human subject were conducted. The real prototype presented positive results on the EMG monitoring, showing the impact of ergonomic design on the smart garment. The EMG system was tested and presented good results, especially in regular movements. However, the system still needs to be improved in order to get a better signal when it comes to movements without pauses.
\end{abstract}

Keywords: EMG $\cdot$ Fit $\cdot$ Vital monitoring $\cdot$ Textile electrode

\section{Introduction}

Surface electromyography is a technique that records the electrical activity of muscles in a non-invasive way. The sEMG electrodes conduct biopotentials generated by the exchange of ions through the membranes of muscle fibers due to muscle contraction and relaxation [1,2] to an appropriate signal conditioning system. This technology has been broadly utilized in muscle activity assessing of athletes and patients $[3,4]$. In sports, EMG signal monitoring has potential benefits such as controlling repetitions, checking muscular fatigue, supporting the development of body awareness, tracking athlete's performance, among others [3,5].

Although the possibility of remote transmission of EMG signals exists, the acquisition method is restrictive and uncomfortable to be conducted in out-of-lab settings. Thus, in recent years, many efforts have been developed by manufacturers and researchers aiming to incorporate this technology in clothes [6-8].

The present study reports the development of a smart garment, more specifically, a long sleeve t-shirt with embedded ECG (Electrocardiography) and EMG electrodes. Initially, two prototypes for cardiac and muscle activity monitoring were developed 
and reported. However, the ECG and EMG systems were negatively impacted by the movement of the arms during use $[9,10]$.

Considering that a perfect fit is of paramount relevance in this kind of smart garment, contributing to the proper operation of the electronic system integrated into the clothing, new prototypes were developed considering the ergonomic aspects of the design. The fit is essential to control the contact, stability, and positioning of electrodes on the user's body and skin [11].

The development and validation of the t-shirt design was aided by tools such as a body scanner and a 3D CAD software. These tools improve the efficiency and effectiveness of the design process and are alternatives to traditional patterning methodologies, expanding the exploration of new project possibilities $[12,13]$.

The main objective of this article is to present the redesign and development process of an ergonomic sportswear garment - a long-sleeve t-shirt - that integrates textile sEMG (Surface Electromyography) electrodes for unobtrusive, real-time monitoring of a person's activity and physiological data.

\section{Materials and methods}

In the development of the smart garment for EMG monitoring, design guidelines were focused on the fit and ergonomic-functional capacity of clothing, as well as on the improvement of contact and stabilization of the shirt to the body to create a better performance environment for electronic monitoring.

The design process was divided into ergonomic design conception, virtual try-on, and tests on a real prototype. For the design, the surface extraction of the 3D digital body obtained using 3D-2D flattening technology was used, through a 3D CAD software, Clo 3D version 4.1. After this, a dynamic virtual try-on analysis, exploring the different positions of the sensors, was performed to develop a virtual prototype.

Subsequently, a physical prototype was developed, using conventional and technical textiles. A Jersey fabric, using $95 \%$ polyamide and $5 \%$ elastane, was used for the t-shirt base, Figure 1. A technical conductive knit Shieldex Medtex P180+B was uses as electrode and for conductive paths. For those, the knit was coated with a thermoplastic film (Bemis Exofliex 3900) and reinforced with TPU. The bonding conditions were: $130^{\circ}$, at $5.5 \mathrm{bar}$, for 20 seconds. The electrodes have a rectangular shape $(5 \mathrm{~cm} \times 3 \mathrm{~cm})$, as shown in Figure 2. The BITalino Board kit, and Opensignals software were used to carry out the experiments. A volunteer was selected to participate in the experiments.

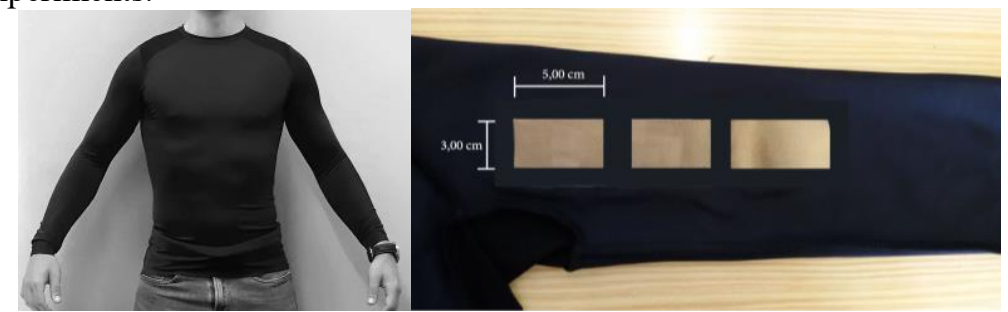

Fig. 1 and 2. T-shirt prototype at use and the electrode design positioned in the sleeve. 
The acquisition procedure was delimited by the repetition of movements related to the muscles studied: biceps, triceps, and chest. The exercises performed were respectively, Dumbbell bicep curls, Kickback, and Push up, and each one was repeated five times by the volunteer. The tests were performed with the t-shirt worn on dry skin and no additional support system for the electrodes was used, ensuring that only the pressure of the t-shirt on the body was applied.

\section{Results and Discussion}

The first ergonomic pattern was designed using a flattening tool available in the 2D3D Cad software Clo $3 D$. This tool creates "a flattenable mesh surface, which is a polygonal mesh surface that can be unfolded into a planar patch without stretching any polygon" [14]. The pattern flattening was done with the delimitation of lines directly over the body model. The tool helped the perception of the ease values necessary in different body postures. The results served as a reference for the design of the final pattern.

Furthermore, the 3D Cad software enabled the try-on prototype tests in dynamic postures. The available avatar was customized according to the volunteer's body measurements, and four body positions were pre-defined as follows: Position 01 body rest (initial); Position 02 - bending elbows; Position 03 - up arms open, and Position 04 - body rest (final). Figure 3 presents the digital try-on test in the four dynamic postures. Additionally, the digital knit was parameterized using the weight, thickness, bend and stretch behavior data from the real knit.

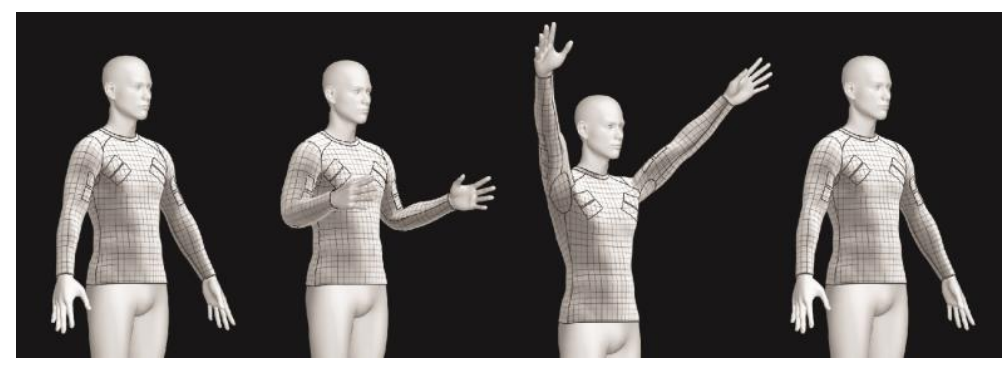

Fig. 3. Digital try-on test in dynamic postures

The results showed the most stable t-shirt zones, contributing to further defining the electrode locations. Figure 4 shows the overlapped images of the t-shirt in Position 1 and Position 4, after the body's movements. 


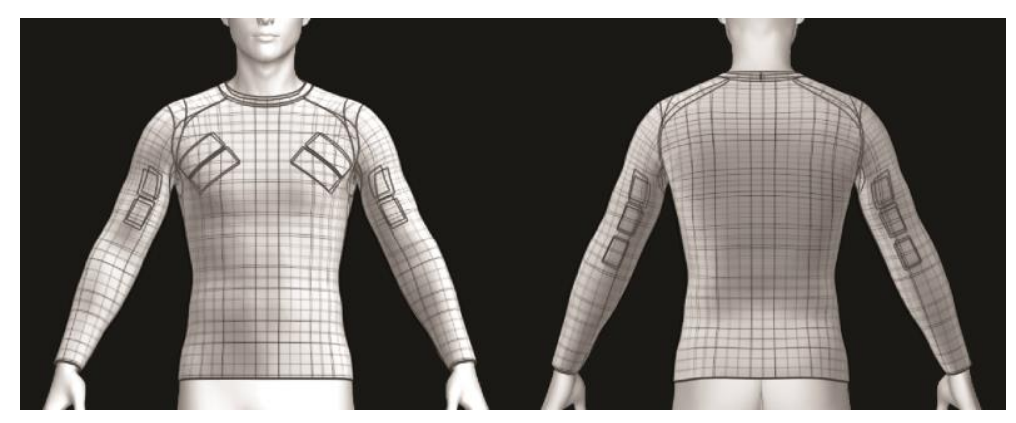

Fig. 4. T-shirt overlap in positions 1 and 4.

The virtual prototype presented good fit performance, despite a small variation of the t-shirt position on the body. But the instability impact was limited way on the electrode zone. The actual prototype proof presented a good fit, free body movement, and stability to the volunteer's body. However, in the area of the shoulders, chest, and upper back still some instability conditioned to the shoulder's movement. A complementary study of localized compression and ergonomic cut will be required for the overall stability of the t-shirt.

Figures 5 to 6 show the EMG signal obtained by Biceps, Triceps, and chest muscles.

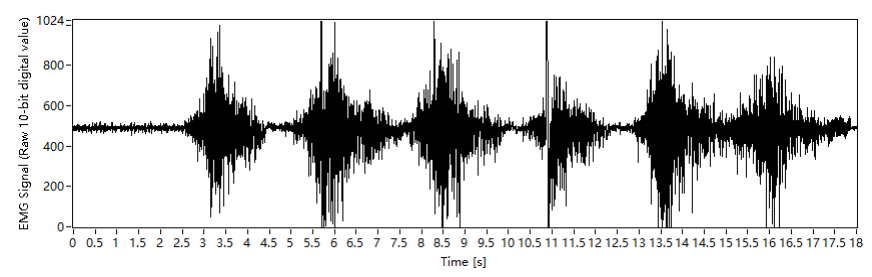

Fig. 5. EMG signal, Muscle: Biceps, Exercise: Dumbbell bicep curls; Repetitions: Six; Weight: $1.0 \mathrm{~kg}$.

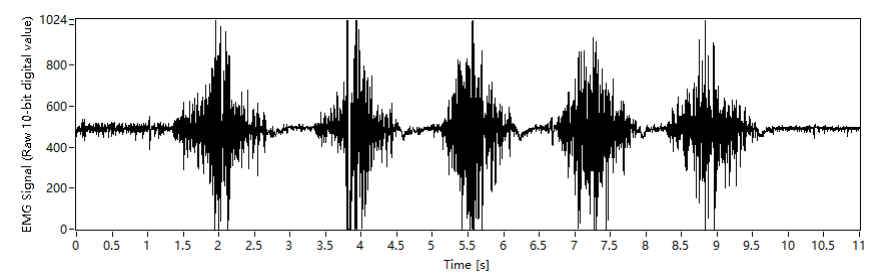

Fig. 6. EMG signal, Muscle: Triceps, Exercise: Kickback; Repetitions: five; Weight: 1.0kg. 


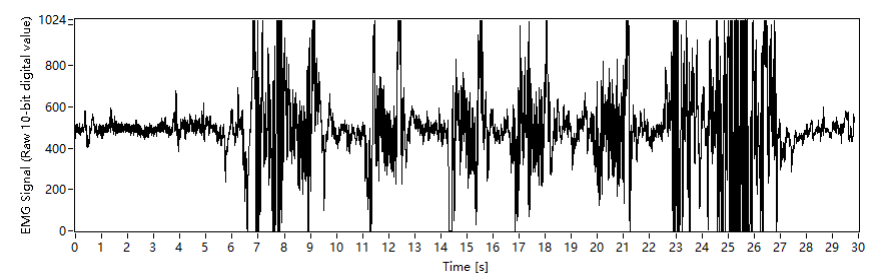

Fig. 7. EMG signal, Muscle: Chest, Exercise: Push up; Repetitions: six.

The electromyographic signals acquisition results can be considered very positive, especially the acquisitions related to biceps and triceps muscles. The waves captured were sharp and with low noise. The graphs showed clearly the muscle's activity and rest moments. These results are promising for future aplications such as monitoring in sports and rehabilitation.

It was also possible to capture the eletric chest signals during pushups exercise. It was possible to identify the peaks of the exercise, but with a higher level of noise than other acquisitions. The noise may be related to the instability of the t-shirt, previously identified. The first and last spikes indicate the beginning and the end of the exercise that involved complementary body movements for the beginning and end of the Arm flexion exercise. The remainder of the exercise graph shows greater homogeneity in the signals, even with the presence of noise.

\section{Conclusions}

In this paper, the ergonomic design developed a smart garment, and EMG measurements result from the t-shirt was reported.

The 3D Cad software supported pattern design and virtual prototypes tests. This assisted the development of a fit and stable t-shirt,according to the smart garment demands. However, there are still instability points on the t-shirt. Future studies will be necessary to achieve solutions to render the electrode positioning less sensitive to body movements.

The BITalino device was used to carry out the experiments, but a device that follows the aesthetic and functional requirements of the garments shall be designed.

Finally, we consider that the ergonomic pattern, from the perspective of the demands of a smart garment, increases the chances of success in future applications of other types of wearable sensors.

\section{Acknowledgment}

This work is financed by Project "Deus ex Machina", NORTE-01-0145-FEDER000026, funded by CCDRN, through Sistema de Apoio à Investigação Cientifica e Tecnológica (Projetos Estruturados I\&D\&I) of Programa Operacional Regional do Norte, from Portugal 2020 and by Project UID/CTM/00264/2019 of 2C2T - Centro de Ciência e Tecnologia Têxtil, funded by National Founds through FCT/MCTES". We also want to thank colleagues Ricardo Moreira for testing the shirt on his body and André Paiva for the knowledge shared with the team. 


\section{References}

1. De la Peña S, Polo A, Robles-Algarín C.: Implementation of a Portable Electromyographic Prototype for the Detection of Muscle Fatigue. Electronics. 619, 2-15 (2019)

2. Jordanić M, Rojas-Martínez M, Mañanas M, Alonso J, Marateb H.: A Novel Spatial Feature for the Identification of Motor Tasks Using High-Density Electromyography. Sensors. 17(7),1597 (2017)

3. MacLean KFE, Dickerson CR.: Kinematic and EMG analysis of horizontal bimanual climbing in humans. J Biomech [Internet]. 2019 May 22

4. Kim H, Lee J, Kim J.: Electromyography-signal-based muscle fatigue assessment for knee rehabilitation monitoring systems. Biomed Eng Lett.8(4), 345-53 (2018)

5. Trindade TB, de Medeiros JA, Dantas PMS, de Oliveira Neto L, Schwade D, de Brito Vieira WH, et al.: A comparison of muscle electromyographic activity during different angles of the back and front squat. Isokinet Exerc Sci.Pre-press, 1-8 (2019).

6. Taelman J, Adriaensen T, van der Horst C, Linz T, Spaepen A.: Textile Integrated Contactless EMG Sensing for Stress Analysis. In: 29th Annual International Conference of the IEEE Engineering in Medicine and Biology Society. ,pp. 3966-9. IEEE Press, New York (2007)

7. Finni T, Hu M, Kettunen P, Vilavuo T, Cheng S.: Measurement of EMG activity with textile electrodes embedded into clothing. Physiological Measurement. 28(11) (2007)

8. Manero RBR, Shafti A, Michael B, Grewal J, Fernandez JLR, Althoefer K, et al.: Wearable embroidered muscle activity sensing device for the human upper leg. In: 38th Annual International Conference of the IEEE Engineering in Medicine and Biology Society (EMBC).. pp.6062-6065. IEEE Press, New York (2016) 9. Paiva A, Catarino A, Carvalho H, Postolache O, Postolache G, Ferreira F.: Design of a long sleeve t-shirt with ECG and EMG for athletes and rehabilitation patients. In: Machado J., Soares F., Veiga G. (eds) Innovation, Engineering and Entrepreneurship. HELIX 2018. Lecture Notes in Electrical Engineering, vol 505, pp. 244-250. Springer (2019)

10. Paiva A, Ferreira F, Catarino A, Carvalho M, Carvalho H.: Design of smart garments for sports and rehabilitation. In: IOP Conf Ser Mater Sci Eng. 459(1) (2019)

11. Harms H, Amft O, Troster G.: Influence of a loose-fitting sensing garment on posture recognition in rehabilitation. In: Proceedings IEEE-BIOCAS Biomedical Circuits and Systems Conference, pp. 353-356. IEEE Press, New York (2008)

12. Hernández N.: Does It Really fit? Improve, find and evaluate garment fit Vol. 25, Stema Specialtryck. [Sweden]: University of Borås; 2018.

13. Fan J, Yu W, Hunter L.: Clothing appearence and fit: Science and Technology. Woodhead Publishing in Textiles, Cambridge (2004)

14. Zhang Y, Wang CCL, Ramani K.: Optimal fitting of strain-controlled flattenable mesh surfaces. Int J Adv Manuf Technol, pp.2873-2887. Springer-Verlag, London (2016)2016;87(9-12):2873-87. 